\title{
Potential feedback control for the power control in LTE
}

\author{
Dat-Duong Phan, Emmanuel Moulay, Patrick Coirault, Frédéric Launay and Pierre Combeau
}

\begin{abstract}
This article deals with the study of uplink (from the mobile phone to the base station) transmitted power control in Long Term Evolution (LTE). It provides a nonlinear Potential Feedback Control (PFC) for SISO discrete-time systems with input delays and disturbances by using the Lyapunov theory and the Artstein transform. It is an original approach in which a stabilization problem of a linear SISO system with a constraint on the state space is transformed into a problem of nonlinear stabilization. We use this strategy of stabilization for the uplink transmission power control in LTE.
\end{abstract}

Index Terms-Potential feedback control, Lyapunov function, discrete-time systems, input delay, LTE.

\section{INTRODUCTION}

The uplink power control is an important task for mobile phone communication systems. It improves the Quality of Service (QoS) in mobile telephony, and more specifically for the new communication standard LTE. On one hand, it sets up a power controller on mobile phones in order to ensure that the Signal to Interference plus Noise Ratio (SINR) stays above a limit below which the communication is interrupted. On the other hand, it consists of minimizing the overall transmitted power in a cell in order to minimize the interferences between users and to maximize the battery life of mobile phones.

Since several years, the interest in wireless cellular networks has stimulated the investigation of several strategies to solve the uplink power control problem [1], [2], [3], [4], [5], [6], [7]. The Transmission Power Control (TPC) strategy, which is an algorithm, has been developed for the new LTE standard [8], [9], [10], [11]. Moreover, the uplink power control in LTE can be modeled as a problem of stabilization of an input delay discrete-time system with a constraint on the state space. So, a suitable strategy for the power control in LTE is the Model Predictive Control (MPC) for a discrete-time system taking into account a constraint on the state space variable [12], [13]. In this article, we provide a new simple feedback control strategy for a SISO system as an alternative solution to the TPC and MPC strategies. It is an original approach in which the stabilization problem of a linear SISO system with a constraint on the state space is transformed into a problem of nonlinear stabilization involving a potential controller. The potential control has essentially been used in robotics to

D. Phan and P. Coirault and F. Launay are with LIAS (EA 6315), École Nationale Supérieure d'Ingénieurs de Poitiers, France (email: dat.duong.phan@univ-poitiers.fr; patrick.coirault@univ-poitiers.fr; frederic.launay@univ-poitiers.fr)

E. Moulay and P. Combeau are with XLIM-SIC (UMR CNRS 7252), Université de Poitiers, France (email: emmanuel.moulay@univ-poitiers.fr, pierre.combeau@univ-poitiers.fr) avoid collisions with obstacles [14], [15]. To the best of our knowledge, this is the first time that a PFC is used for a discrete-time system. The term "potential" is used to refer to the Coulomb potential which is an impassable barrier. A PFC is a nonlinear feedback control ensuring that the system output is always higher than a lower limit. Applying this approach in LTE, it leads to the easy adjustment of two parameters for each mobile phone. The main difficulty of this strategy of stabilization is due to the nonlinearity of the potential term which implies the use of the Lyapunov theory. In order to take into account the mobility of mobile phones, an input delay which denotes the propagation time and computation time is added. So, we use the Artstein transform for discretetime systems to deal with this input delay [16], [17].

The paper is organized as follows. The uplink power control in LTE is described in section II. Then, the PFC for discretetime systems is developed in section III. It is applied and simulated for a cellular network in LTE in section IV and a comparison with the TPC and MPC strategies is given. Finally, a conclusion is addressed in section $\mathrm{V}$.

\section{UPLINK POWER CONTROL IN LTE}

\section{A. Modeling of a transmission channel}

In this article, we are interested in the uplink channel between a mobile phone denoted by $k$ and a base station of the cell denoted by $i$. In urban environments, the channel gain of a radio link can be modeled by three components: path-loss, log-normal shadowing, and multi-path fading. The gain of the channel is defined as

$$
g_{i k}(n)=\frac{\gamma_{t} \gamma_{r} \lambda^{2}}{(4 \pi)^{2} d_{i k}^{2} \ell} \cdot 10^{0.1 \delta(n)} \cdot|r(n)|^{2}
$$

where $n$ is the sample time. The term $\frac{\gamma_{t} \gamma_{r} \lambda^{2}}{(4 \pi)^{2} d_{i k}^{2} \ell}$ is the far field gain with $\gamma_{t}$ the transmitting antenna gain, $\gamma_{r}$ the receiving antenna gain, $d_{i k}$ the distance between the transmitter of the cell $i$ and the receiver $k$ in meters, $\ell$ the system loss factor not related to propagation $(\ell \geq 1)$ and $\lambda$ the wavelength in meters [18]. The term $10^{0.1} \delta(n)$ is used to model large-scale lognormal shadowing where $\delta(n)$ is a normal Gaussian random variable [19]. The term $|r(n)|^{2}$ is used to model Rayleigh fading where $r(n)$ is the random variable that has a Rayleigh distribution [18].

The received SINR is the ratio of the received signal power to the power of the interference plus noise within the bandwidth of the transmitted signal. The SINR is commonly used in wireless communication as a way to measure the quality of transmission. In LTE, the use of Orthogonal Frequency 
Division Multiple Access (OFDMA) and Single Carrier Frequency Division Multiple Access (SC-FDMA) avoids the intra-cell interferences [20], [10]. Therefore, after frequency synchronization, only the inter-cell interferences affect the SINR in the model.

Notation 1: The lower case is used for the linear domain and the upper case for the logarithm domain in $\mathrm{dB}$ of the parameters. For instance, $x_{i k}$ stands for the SINR in linear domain and $X_{i k}$ stands for the SINR in the logarithm domain and we have $X_{i k}=10 \log _{10} x_{i k}$.

The SINR of the mobile phone $k$ at the base station $i$ is given by

$$
x_{i k}(n)=\frac{g_{i k}(n) \cdot p_{i k}(n)}{\sum_{\ell \neq k} g_{i \ell}(n) \cdot p_{i \ell}(n)+\sigma_{i k}^{2}(n)}
$$

where $p_{i \ell}$ and $g_{i \ell}$ are respectively the transmitted power and channel gain from the mobile phone $\ell$ belonging to the neighboring base stations of $i, \sigma_{i k}^{2}$ the thermal noise affecting the channel between the mobile phone $k$ and the base station of the cell $i$. The term $\sum_{\ell \neq k} g_{i \ell}(n) \cdot p_{i \ell}(n)$ corresponds to the inter-cell interferences. As it was explained above, the intracell interferences in LTE are negligible. So, we can rewrite (2) as follows

$$
x_{i k}(n)=\frac{g_{i k}(n) \cdot p_{i k}(n)}{i_{i k}(n)+\sigma_{i k}^{2}(n)}
$$

where $i_{i k}=\sum_{\ell \neq k} g_{i \ell}(n) \cdot p_{i \ell}(n)$ are the inter-cell interferences.

\section{B. Uplink PUSCH power control}

A presentation of the LTE standard and the LTE physical layer is given in [21]. There are several kinds of uplink physical channels such as:

- the Physical Uplink Control Channel (PUCCH) which is used to support the transmission of acknowledgment, scheduling requests, the Channel Quality Indicator (CQI) and the Sounding Reference Symbol (SRS) [11, Section 5.1.3] which is used at the base station for the channel estimation;

- the Physical Uplink Shared Channel (PUSCH) which is the main physical channel used for the data transmission and paging information.

We are interested in the power control of the PUSCH channel which is the main physical channel of the physical layer. In LTE, the power control is computed at the base station [11, Page 11].

The setting of the User Equipment (UE) (which can be for instance a mobile phone) transmission power for a $\mathrm{PUSCH}$ transmission is defined as follows [11]

$$
\begin{aligned}
P_{P U S C H, c}(j) & =\min \left(P_{C M A X, c}, 10 \log _{10} M_{P U S C H, c}(j)\right. \\
& +P_{O, P U S C H, c}(\beta)+\alpha_{c}(\beta) \cdot P L_{c}(j) \\
& \left.+\Theta_{T F, c}(j)+F_{c}(j)\right)
\end{aligned}
$$

where $j$ is the number of the subframe, $\beta=0,1,2$ a specific parameter in LTE, $P_{C M A X, c}$ the maximum transmit power for serving cell $c, M_{P U S C H}(j)$ the measured bandwidth in number of resource blocks valid for subframe $j$ and serving cell $c, P_{O, P U S C H, c}(\beta)$ the parameter provided by higher layers which has a cell-specific and nominal part expressing the power to be contained in one physical resource block, $\alpha_{c}(\beta) \in[0,1]$ a cell-specific parameter allowing the compensation of partial pathloss and provided by higher layers, $P L_{c}$ the downlink pathloss estimate calculated at the UE for serving cell $c, \Theta_{T F, c}$ the UE specific parameter depending on the chosen Modulation and Coding Scheme (MCS), $F_{c}(j)$ a current $P U S C H$ power control adjustment state for serving cell $c$ defined by

$$
F_{c}(j)=F_{c}(j-1)+\Delta_{P U S C H}\left(j-K_{P U S C H}(j)\right)
$$

where $\Delta_{P U S C H}\left(j-K_{P U S C H}(j)\right)$ is a correction value, also referred to as a Transmit Power Command (TPC) which was signaled on subframe $\left(j-K_{P U S C H}(j)\right), K_{P U S C H}(j)$ is the downlink delay corresponding to the propagation time and the calculation time of the processor, $K_{P U S C H}(j) \in\{4, \cdots, 7\}$. $P_{C M A X, c}$ depends on the maximum allowed power configured by higher layers and the maximum UE power class.

In the remainder of this paper, we will replace the number of the subframe $j$ by the time variable $n$ because frames and subframes are sent successively in time. The indexes $c, P U S C H$ and $T F$ which are specific to the LTE standard will be omitted. For instance, $F_{c}(i)=F_{c}(i-1)+\Delta_{P U S C H}\left(i-K_{P U S C H}(i)\right)$ becomes

$$
F(n)=F(n-1)+\Delta(n-K)
$$

In the linear domain, the SINR is defined by equation (3). Let us define the pathloss $p l$ by $p l_{i k}(n)=\frac{1}{g_{i k}(n)}$ and $i_{o} t_{i k}(n)=\frac{i_{i k}(n)+\sigma_{i k}^{2}(n)}{\sigma_{i k}^{2}(n)}$. We can rewrite equation (3) as follows

$$
x_{i k}(n)=\frac{p_{i k}(n)}{p l_{i k}(n) \cdot \sigma_{i k}^{2}(n) \cdot i_{o} t_{i k}(n)} .
$$

We obtain the equation of the SINR in the logarithm domain

$$
X_{i k}(n)=P_{i k}(n)-P L_{i k}(n)-\Sigma_{i k}^{2}(n)-I_{o} T_{i k}(n) .
$$

In the linear domain, the interference term $i_{i k}$ and the thermal noise $\sigma_{i k}^{2}$ are measured at the base station [22]. So, we have the terms $\Sigma_{i k}^{2}(n)$ and $I_{o} T_{i k}(n)$. The term $P_{i k}$ is known at the base station and the term $P L_{i k}$ is estimated with the SRS. Then, we have the SINR $X_{i k}$ at the base station by using (7).

Now, we develop a state space model for the uplink power control in LTE. In the following, and for simplicity, we will omit the indexes $i$ and $k$ in the equations and $X(n)$ and $P(n)$ stands for $X_{i k}(n)$ and $P_{i k}(n)$. The $U$ stands for $\triangle_{P U S C H}$ defined in the previous section. Therefore, equation (6) becomes

$$
F(n)=F(n-1)+U(n-K)
$$

We use the $z$-transform from equation (8) and we obtain

$$
F(z)=\frac{z^{-K}}{1-z^{-1}} U(z) .
$$

In the sequel, and for clarity, we will omit the saturation term $P_{C M A X}$ given in (4) but it will be taken into account in the simulations of section IV with the use of a saturation bloc 
(see the figure 1). However, no elaborate strategy is provided to treat this saturation. From equation (4), we have

$$
P(z)=W_{1}(z)+F(z)=W_{1}(z)+\frac{z^{-K}}{1-z^{-1}} U(z)
$$

where $W_{1}(z)=10 \log _{10} M(z)+P_{0}(z)+\alpha(j) \cdot P L(z)+$ $\Theta(z)$. As the round-trip delay $M$, which is the sum of the downlink and the uplink delays, can be considered constant (see [23], [24]), the uplink delay is equal to $M-K$ with $K \in\{4, \cdots, 7\}$. From equation (7) and taking into account the propagation delay, we approximate

$$
X(z)=\left(W_{2}(z)+P(z)\right) z^{-(M-K)}
$$

where $W_{2}(z)=-P L(z)-\Sigma^{2}(z)-I_{o} T(z)$. We substitute equation (10) in equation (11) and we obtain

$$
X(z)=\frac{z^{-M}}{1-z^{-1}} U(z)+\left(W_{1}(z)+W_{2}(z)\right) z^{-(M-K)} .
$$

Multiplying both sides of the equation (12) by $\left(1-z^{-1}\right)$, we get

$\left(1-z^{-1}\right) X(z)=z^{-M} U(z)+\left(W_{1}(z)+W_{2}(z)\right)\left(1-z^{-1}\right) z^{-(M-K)}$.

Now, using the $z$-inverse transform we obtain the following discrete-time system

$$
X(n)=X(n-1)+U(n-M)+H(n-1)
$$

with the known input $H(n-1)=W_{2}(n-(M-K))-W_{2}(n-$ $(M-K)-1)+W_{1}(n-(M-K))-W_{1}(n-(M-K)-1)$ and a constant delay $M-1$ on the control. It is worth noting that the values of $W_{1}$ and $W_{2}$ must be archived in a register to be used in equation (13), the values of $K \in\{4, \cdots, 7\}$ are known.

The round-trip delay is a constant in LTE $M=10 \mathrm{~ms}$ including the calculation time of the processor [23], [24]. The delays on the exogenous known inputs of the system $W_{1}$ and $W_{2}$ are not critical because it only means that data are stored. Let $\hat{H}(n)$ be the estimation of the exogenous input such that $|H(n)-\hat{H}(n)|<\epsilon$. We rewrite (13) as follows

$$
X(n)=X(n-1)+V(n-M)-\hat{H}(n-1)+H(n-1)
$$

where $V(n-M)=U(n-M)+\hat{H}(n-1)$ is a new virtual control variable. It leads to

$$
X(n)=X(n-1)+V(n-M)+D(n-1)
$$

where $D(n-1)=H(n-1)-\hat{H}(n-1)$ is the disturbance on the exogenous input $H(n-1)$.

Let us give the block diagram of the system in figure 1, including a receiver and a transmitter corresponding to a base station and a mobile phone.

\section{PFC FOR DISCRETE-TIME SYSTEMS}

The basic definitions of asymptotic stability and Lyapunov functions for discrete-time systems with disturbances are given in [25, Definitions 2.1 and 2.6]. The Lyapunov theorems for discrete-time systems used in this article are given in [26], [27].

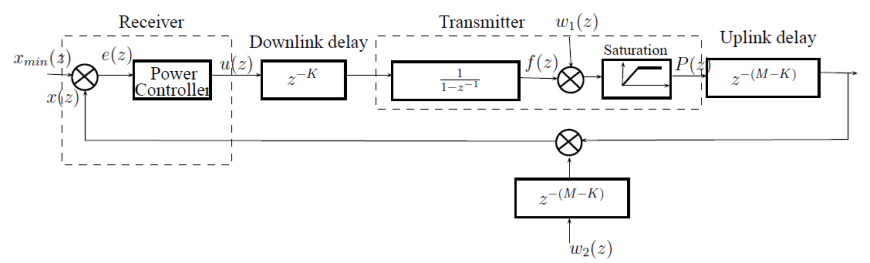

Fig. 1. The closed-loop power control system for each user

In this section, we develop our PFC for SISO systems with disturbances in order to take into account the constraint on the state variable.

Theorem 1: Consider the system given by

$$
x(n+1)=x(n)+u(n)+d(n), \quad n \in \mathbb{N}
$$

where $x(n) \in \mathbb{R}$ is the state, $u(n) \in \mathbb{R}$ the input and $|d(n)| \leq \epsilon$ a bounded disturbance. Let $c \leq 0$, under the initial condition $x(0)>c$ the system (15) with the feedback control

$$
u(n)=k_{1}(x(n)-c)+\frac{k_{2}}{x(n)-c}
$$

with $-\frac{1}{2}<k_{1}<0$ and $k_{2} \geq \frac{\epsilon^{2}}{2\left(2 k_{1}+1\right)}$ is asymptotically stable with respect to the attractive and invariant set $S=\left[\frac{-\epsilon+\sqrt{\epsilon^{2}-4 k_{1} k_{2}}}{-2 k_{1}}+c ; \frac{-\epsilon\left(k_{1}-1\right)+\sqrt{\epsilon^{2}\left(k_{1}-1\right)^{2}-4 k_{1}^{3} k_{2}}}{2}+c\right]$. Moreover, we have $x(n) \geq \frac{-\epsilon+\sqrt{\epsilon^{2}-4 k_{1} k_{2}}}{-2 k_{1}}+c$ for all $n \in$ $\mathbb{N}^{*}=\mathbb{N} \backslash\{0\}$

Proof. We have two possible cases which depend on the sign of the initial condition $x(0)$ of the system (15).

First case: $x(0)>0$. We take $c=0$. Firstly, we seek the equilibrium points of the closed-loop system. With the following input $u(n)=k_{1} x(n)+\frac{k_{2}}{x(n)}$, the system (15) becomes

$$
x(n+1)=\left(k_{1}+1\right) x(n)+\frac{k_{2}}{x(n)}+d(n) .
$$

The positive equilibrium interval is given by

$$
S_{e q}^{+}=\left\{\bar{x}>0: \bar{x}=\left(k_{1}+1\right) \bar{x}+\frac{k_{2}}{\bar{x}}+d(n) ;|d(n)| \leq \epsilon\right\} .
$$

If $\bar{x} \neq 0$ then we have $k_{1} \bar{x}^{2}+d(n) \bar{x}+k_{2}=0$. If $k_{1}<0$ and $k_{2}>0$ then we have a positive root $x^{+}(n)=\frac{d(n)+\sqrt{\Delta}}{-2 k_{1}}$ where $\Delta=d(n)^{2}-4 k_{1} k_{2}$. So, we obtain

$$
S_{e q}^{+}=\left[\frac{-\epsilon+\sqrt{\epsilon^{2}-4 k_{1} k_{2}}}{-2 k_{1}} ; \frac{+\epsilon+\sqrt{\epsilon^{2}-4 k_{1} k_{2}}}{-2 k_{1}}\right] .
$$

We seek the minimum value of $x(n+1)$. From (17), we have

$$
\frac{\partial x(n+1)}{\partial x(n)}=\frac{\left(k_{1}+1\right) x(n)^{2}-k_{2}}{x(n)^{2}}
$$

for $n \in \mathbb{N}$. For $k_{1}>-1$, the roots of (18) are $x(n)=$ $\pm \sqrt{\frac{k_{2}}{k_{1}+1}}$. As $x(0)>0$, we have $x(n+1) \geq \sqrt{4\left(k_{1}+1\right) k_{2}}-$ $d$ for all $n \in \mathbb{N}$. So, the interval of possible minimum values for $x(n+1)$ is given by

$$
S_{\text {min }}=\left[\sqrt{4\left(k_{1}+1\right) k_{2}}-\epsilon ; \sqrt{4\left(k_{1}+1\right) k_{2}}+\epsilon\right] .
$$

The smallest value of $S_{\min }$ is $\min S_{\min }=\sqrt{4\left(k_{1}+1\right) k_{2}}-\epsilon$. We want that $\min S_{\min }>0$, therefore $k_{2}>\frac{\epsilon^{2}}{4\left(k_{1}+1\right)}$. We 
impose $\min S_{\min } \geq \min S_{e q}^{+}$under the conditions $-1<k_{1}<$ $0, k_{2}>\frac{\epsilon^{2}}{4\left(k_{1}+1\right)}$ and $\epsilon>0$. It leads to the following algebraic inequality$$
-\epsilon\left(1+2 k_{1}\right)+\sqrt{\epsilon^{2}-4 k_{1} k_{2}}+2 k_{1} \sqrt{4 k_{2}\left(k_{1}+1\right)} \leq 0 .
$$

A symbolic calculator gives the following sufficient conditions $\epsilon>0,-\frac{1}{2}<k_{1}<0, k_{2} \geq \frac{\epsilon^{2}}{2\left(2 k_{1}+1\right)}$ on $\epsilon, k_{1}$ and $k_{2}$ in order to satisfy the inequality (19). Under these conditions, we have $x(n) \geq \frac{-\epsilon+\sqrt{\epsilon^{2}-4 k_{1} k_{2}}}{-2 k_{1}}$ for all $n \in \mathbb{N}^{*}$ and a relevant minimum value for the attractive and invariant set.

Now we have to prove the stability of the closed-loop system (15), (16). Let us consider the following Lyapunov function

$$
V(n, x(n))=x(n)-x^{+}(n)
$$

for $x(n)>\max S_{e q}^{+}=\frac{\epsilon+\sqrt{\epsilon^{2}-4 k_{1} k_{2}}}{-2 k_{1}}$ and $n \in \mathbb{N}^{*}$. We have

$$
\begin{aligned}
\Delta V(n, x(n)) & =V(n+1, x(n+1))-V(n, x(n)) \\
& =\frac{k_{1} x(n)^{2}+\left(d(n)-x^{+}(n+1)+x^{+}(n)\right) x(n)+k_{2}}{x(n)} .
\end{aligned}
$$

It is easy to see that $\Delta V(n, x(n))<0$ if $x(n)>x^{*}(n)$ where

$$
x^{*}(n)=\left(d(n)-x^{+}(n+1)+x^{+}(n)\right)+\sqrt{\delta}
$$

with

$$
\delta=\left(d(n)-x^{+}(n+1)+x^{+}(n)\right)^{2}-4 k_{1} k_{2} .
$$

We have

$$
\max _{n \in \mathbb{N}^{*}} x^{*}(n)=\frac{-\epsilon\left(k_{1}-1\right)+\sqrt{\epsilon^{2}\left(k_{1}-1\right)^{2}-4 k_{1}^{3} k_{2}}}{2}
$$

and this maximum value is reached for $d(n)=\epsilon, x^{+}(n)=$ $\frac{\epsilon+\sqrt{\epsilon^{2}-4 k_{1} k_{2}}}{-2 k_{1}}$, and $x^{+}(n+1)=\frac{-\epsilon+\sqrt{\epsilon^{2}-4 k_{1} k_{2}}}{-2 k_{1}}$. As the maximum value $\max _{n \in \mathbb{N}^{*}} x^{*}(n)$ is greater than $\max S_{e q}^{+}$for $-\frac{1}{2}<k_{1}<0$, we define the attractive and invariant set by

$$
S_{0}=\left[\frac{-\epsilon+\sqrt{\epsilon^{2}-4 k_{1} k_{2}}}{-2 k_{1}} ; \frac{-\epsilon\left(k_{1}-1\right)+\sqrt{\epsilon^{2}\left(k_{1}-1\right)^{2}-4 k_{1}^{3} k_{2}}}{2}\right]
$$

and we have $\Delta V(n, x(n))<0$ for all $x(n)>\max S_{0}$.

To conclude, we have to prove that $S_{0}$ is invariant. Due to the previous calculus of $S_{\min }$, we have

$$
\min _{x(n) \in S_{0}} x(n+1) \geq \min S_{0} .
$$

Now, let us calculate $\max _{x(n) \in S_{0}} x(n+1)$ and check that $\max _{x(n) \in S_{0}} x(n+1) \leq \max S_{0}$. Due to the variations of the continuous function $x(n+1)$ with respect to the variable $x(n)$ and the intermediate value theorem, we only have to prove that $\left.x(n+1)\right|_{\min S_{0}} \leq \max S_{0}$ and $\left.x(n+1)\right|_{\max S_{0}} \leq \max S_{0}$. It is quite easy but lengthy to check both conditions and this is left to the reader.

By using the Lyapunov theorem for discrete systems given in [25, Theorem 1], we conclude that if $\epsilon>0,-\frac{1}{2}<k_{1}<0$ and $k_{2} \geq \frac{\epsilon^{2}}{2\left(2 k_{1}+1\right)}$ then the system (15) is asymptotically stable with respect to the interval $S_{0}$.

Second case: $x(0) \leq 0$. From equations (15) and (16) we have

$$
x(n+1)=x(n)+k_{1}(x(n)-c)+\frac{k_{2}}{x(n)-c}+d(n) .
$$

Subtracting $c$ in both sides of the equation (20), we get

$$
x(n+1)-c=x(n)-c+k_{1}(x(n)-c)+\frac{k_{2}}{x(n)-c}+d(n) .
$$

Consider the following change of variable

$$
\psi(n)=x(n)-c
$$

that leads to the new system

$$
\psi(n+1)=\left(k_{1}+1\right) \psi(n)+\frac{k_{2}}{\psi(n)}+d(n) .
$$

We take $c<x(0)$, it leads to $\psi(0)>0$. Therefore, we can apply the proof of the first case. We deduce that if $c<x(0)$, $\epsilon>0,-\frac{1}{2}<k_{1}<0$ and $k_{2} \geq \frac{\epsilon^{2}}{2\left(2 k_{1}+1\right)}$ then the system (15) is asymptotically stable with respect to the interval $S$

$$
S=\left[\frac{-\epsilon+\sqrt{\epsilon^{2}-4 k_{1} k_{2}}}{-2 k_{1}}+c ; \frac{-\epsilon\left(k_{1}-1\right)+\sqrt{\epsilon^{2}\left(k_{1}-1\right)^{2}-4 k_{1}^{3} k_{2}}}{2}+c\right]
$$

Remark 1: If the value of $k_{1}$ is close to $-\frac{1}{2}$ then $\max S$ is rather close to $\min S$ and we can ensure a good interval of convergence. If the value of $k_{1}$ is close to zero then $\max S$ is distant from $\min S$ and we cannot ensure a good interval of convergence. Moreover, a small value of $k_{2}$ satisfying the condition $x(n) \geq \frac{-\epsilon+\sqrt{\epsilon^{2}-4 k_{1} k_{2}}}{-2 k_{1}}+c$ for all $n \in \mathbb{N}^{*}$ ensures a small interval of convergence. The stability of the system (15) remains even if there are bounded disturbances which implies a certain robustness.

In order to take into account a constant delay on the input during the transmission in LTE, we develop a new result on the stabilization for SISO systems with disturbances and constant delay on the input involving a potential controller. The proof of this result is based on the discrete-time Artstein transform.

Theorem 2: Consider the system given by

$$
x(n+1)=x(n)+u\left(n-T_{d}\right)+d(n), \quad n \in \mathbb{N}
$$

where $x(n) \in \mathbb{R}$ is the state, $u(n) \in \mathbb{R}$ the input, $T_{d} \in \mathbb{N}^{*}$ the delay and $|d(n)| \leq \epsilon$ a bounded disturbance. Let $c \leq 0$, under the initial condition $x(0)>c$ the system (25) with the feedback control

$$
u(n)=k_{1}(\xi(n)-c)+\frac{k_{2}}{\xi(n)-c}, \quad n \in \mathbb{N}
$$

with $u(n)=0$ for $n<0,-\frac{1}{2}<k_{1}<0, k_{2} \geq \frac{\epsilon^{2}}{2\left(2 k_{1}+1\right)}$ and $\xi(n)=x(n)+\sum_{i=1}^{T_{d}} u(n-i)$, is asymptotically stable with respect to the attractive and invariant set $S_{d}=\left[\min S_{d} ; \max S_{d}\right]$ where $\min S_{d}=\frac{-\epsilon+\sqrt{\epsilon^{2}-4 k_{1} k_{2}}}{-2 k_{1}}+c-T_{d} \epsilon$ and $\max S_{d}=$ $\frac{-\epsilon\left(k_{1}-1\right)+\sqrt{\epsilon^{2}\left(k_{1}-1\right)^{2}-4 k_{1}^{3} k_{2}}}{2}+c+T_{d} \epsilon$. Moreover, we have $x(n) \geq \frac{-\epsilon+\sqrt{\epsilon^{2}-4 k_{1} k_{2}}}{-2 k_{1}}+c-T_{d} \epsilon$ for all $n \geq T_{d}$.

Proof. The discrete-time Artstein transform allows us to transform a linear discrete-time controlled system having a constant input delay into a system without delay. This transformation is presented in [16], [17] for MIMO discrete-time systems. Applying the Artstein transform to the system (25), we obtain the following system without delay

$$
\xi(n+1)=\xi(n)+u(n)+d(n)
$$


where

$$
\xi(n)=x(n)+\sum_{i=1}^{T_{d}} u(n-i) .
$$

We have that the state $\xi($.$) of the system (27) is asymptot-$ ically stable with respect to the interval $S$ with the feedback control (16) due to theorem 1. By using the Artstein transform, we deduce that $x($.$) is asymptotically stable with respect to a$ set $S_{d}$ we have to determine. From equation (27) we deduce

$$
\sum_{i=1}^{T_{d}} u(n-i)=\xi(n)-\xi\left(n-T_{d}\right)-\sum_{i=1}^{T_{d}} d(n-i+1),
$$

and from equations (28), (29) we obtain

$$
x(n)=\xi\left(n-T_{d}\right)+\sum_{i=1}^{T_{d}} d(n-i+1) .
$$

As $d($.$) is bounded by assumption and \xi($.$) is bounded due$ to theorem 1 , then the state $x($.$) is also bounded due to (30).$ Therefore, there exists a minimum value $x_{\min }$ such that the state $x($.$) is always higher than x_{m i n}$. From equation (30), we deduce

$$
x_{\text {min }}=\xi_{\text {min }}-T_{d} \epsilon
$$

where $\xi_{\text {min }}=\frac{-\epsilon+\sqrt{\epsilon^{2}-4 k_{1} k_{2}}}{-2 k_{1}}$ due to theorem 1 . This minimum value $x_{\min }$ is also the minimum value $\min S_{d}$ of the attractive and invariant set of system (25). Moreover, it is easy to check that the maximum value $\max S_{d}$ of the attractive and invariant set of system (25) satisfies

$$
\max S_{d}=\max S+T_{d} \epsilon .
$$

So, we have found the attractive and invariant set $S_{d}$. As we have $\xi(n) \geq \xi_{\min }$ for all $n>0$, then we have $x(n) \geq x_{\min }$ for all $n>T_{d}$. This concludes the proof.

\section{Simulation Results}

We now provide the simulations of the SINR in LTE for the modeling presented in section II. For the simulations, we suppose that there is an estimated error of $1,1 \mathrm{~dB}$ on the exogenous input $H(n)$. The evaluation of this value depends on the quality of the channel gain estimator. In the figure $1, x_{\min }(z)$ is a minimal SINR corresponding to a highest acceptable Block Error Rate (BLER) for communication between transmitter and receiver. The figure 2 allows us to find the minimum value of the SINR denoted by SINR $_{\min }$. The maximum BLER given by the LTE standard is $10^{-1}$. For a given modulation rate $r$, the relation between the BLER and the SINR is illustrated by the figure 2. We suppose for the simulations that the SINR is equal to the SNR, as in [28]. We choose the 64QAM modulation and $r=2 / 3$ for our simulation. We find the value of $\operatorname{SINR}_{\min }=14,37 \mathrm{~dB}$. For the simulations, we suppose that the distance between the mobile phone and the base station varies between $18,80 \mathrm{~m}$ and $342,67 \mathrm{~m}$, so the $P L$ varies between $63,93 \mathrm{~dB}$ and $89,17 \mathrm{~dB}$. We assume the values of $P_{0}(n)=-78 \mathrm{dBm}, M(n)=50$, $\alpha(j)=0,8$ given in [29]. Moreover, we suppose that $I_{o} T(n)$ varies between $3 \mathrm{~dB}$ and $7 \mathrm{~dB}$ and $\Sigma^{2}(n)$ varies between

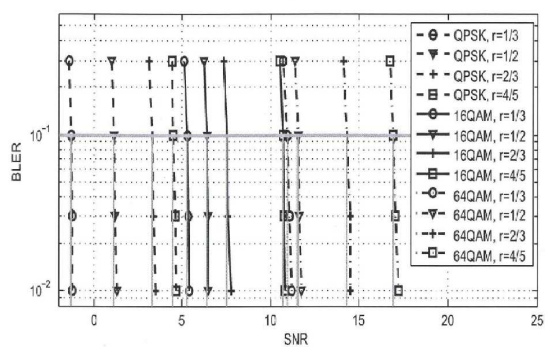

Fig. 2. The BLER versus the SINR $(\mathrm{dB})$ for a different modulation types and rates $r$ [21]

$-110 \mathrm{dBm}$ and $-120 \mathrm{dBm}$. The coherence time of the channel is given by

$$
T_{c}=\sqrt{\frac{9}{16 \pi f_{m}^{2}}}=\frac{0.423}{f_{m}}
$$

where $f_{m}=\frac{v}{\lambda}$ is the maximum Doppler shift with $v$ the velocity [18]. In LTE, the carrier frequency $f_{c}=\frac{c}{\lambda}=1.8 \mathrm{GHz}$. The control sample time $T_{e}$ at which we refresh the feedback control cannot exceed the coherence time of the channel. We apply our feedback control (16) with a sample time of $50 \mathrm{~ms}$ for a mobile phone which moves slowly with a velocity of $5 \mathrm{~km} . \mathrm{h}^{-1}$ in a cell having a radius of 500 meters. So, the input delay which is much lower than the sample time is neglected. Due to the theorem 1, we choose the value of $k_{1}=-0.49$, $c=0$ and we impose $\operatorname{SINR}_{\min }=\min S$. Then, the minimum value of $k_{2}$, being an integer such as $x(n) \geq 14,37 \mathrm{~dB}$ for all $\mathrm{n}$ $\mathbb{N}^{*}$, is given by $k_{2}=117$. The figure $3 a$ presents the evolution of the SINR of a mobile phone which moves with a velocity of $5 \mathrm{~km} . \mathrm{h}^{-1}$ with $T_{e}=50 \mathrm{~ms}$. We can see in the figure $3 a$ that the SINR is always higher than the $\operatorname{SINR}_{\text {min }}$ and lower than the upper bound of the attractive and invariant set $S$ in the steady-state with the PFC. So, the system is asymptotically stable with respect to $S$. The term $\frac{k_{2}}{x(n-1)}$ allows the SINR to be higher than the SINR min $_{\text {in }}$ in one sample time.

Now, suppose that the velocity of the mobile phone is $260 \mathrm{~km} . \mathrm{h}^{-1}$ then $T_{c}=1 \mathrm{~ms}$. So, we chose the sample time $T_{e}=1 \mathrm{~ms}$ which is a classical sample time in LTE for the power control [20]. In order to apply the power control, we have to take into account the input delay which is then higher than the sample time. Due to the theorem 2, we have $\xi_{\min }=x_{\min }+\frac{M-1}{T_{e}} \epsilon=24.27 \mathrm{~dB}$. We chose the values of $k_{1}=-0.49, c=0$ and we impose SINR $_{m i n}=\min S_{d}$. We deduce the value of $k_{2}=316$. The figures $3 b, 3 c$ correspond to the evolution of the SINR of a mobile phone which moves with a velocity of $260 \mathrm{~km} . \mathrm{h}^{-1}$ with $T_{e}=1 \mathrm{~ms}$. We can see in the figure $3 b$ that, by using the PFC (26), the system with input delay is above the SINR $_{\text {min }}$ after 9 steps which corresponds to $\frac{M-1}{T_{e}}$ and is asymptotically stable. A comparison with the TPC and the MPC (having a minimal prediction horizon of 1) and taking into account the same perturbation of $1,1 \mathrm{~dB}$ is also highlighted in the figures $3 a, 3 b$. We can see that the efficiency of both strategies is quite similar. We have to take into account the input delay in the PFC strategy when we have a high mobility. Indeed, we see in the figure $3 c$ that the SINR is not always higher than the SINR ${ }_{\text {min }}$ if we do not apply the 
PFC (26) of the theorem 2 but the PFC (16) of the theorem 1 to the system (14).

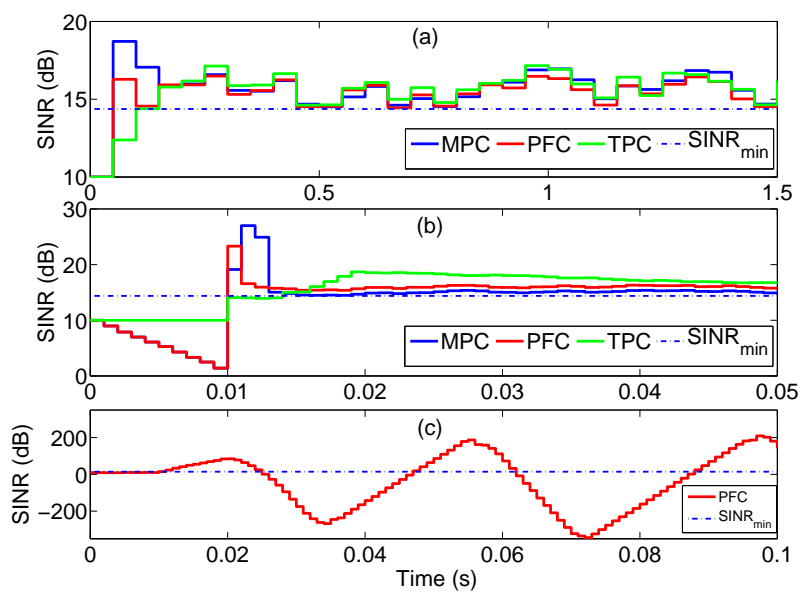

Fig. 3. (a) SINR with a velocity of $5 \mathrm{~km} . \mathrm{h}^{-1}$; (b) (c) SINR with a velocity of $260 \mathrm{~km} \cdot \mathrm{h}^{-1}$ with and without Artstein transform

Moreover, we see in the figure 4 that the execution time, for a simulated time period of the system of $10 \mathrm{~s}$, of the PFC is always shorter than the one of the TPC and the MPC. This is an advantage for the real-time computing due to the fact that we have no prediction to do with the PFC. The figure 4 highlights that the real-time computing can become critical because the number of mobile phones can be very high in a cellular network.

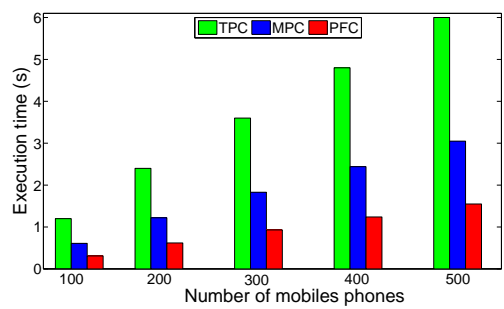

Fig. 4. Execution time for a simulated time period of the system of $10 \mathrm{~s}$

\section{CONCLUSION}

In this article, we have developed a new feedback control strategy for discrete-time systems having a constraint on the state space variable and an input delay. This strategy is based on a nonlinear PFC. It is well suited for the power control of a wireless cellular network due to the constraints on the minimum value of the SINR imposed by the telecommunication standards and on the real-time computing. In our application in LTE, it ensures that the SINR is always higher than a given minimum value. As future research lines, it will be interesting to extend the PFC to MIMO systems.

\section{REFERENCES}

[1] T. Charalambous, "Power control in wireless ad hoc networks: Stability and convergence under uncertainties," in Optimization, Simulation, and Control, ser. Springer Optimization and Its Applications. Springer, 2013, pp. 143-174.
[2] T. Frantti and P. Mähönen, "Adaptive fuzzy power control for WCDMA mobile radio systems," Control Engineering Practice, vol. 9, no. 9, pp. 947-959, 2001.

[3] F. Gunnarsson and F. Gustafsson, "Control theory aspects of power control in UMTS," Control Engineering Practice, vol. 11, no. 10, pp. 1113-1125, 2003.

[4] S. Lasaulce and H. Tembine, Game Theory and Learning for Wireless Networks: Fundamentals and Applications. Academic Press, 2011.

[5] K. Shoarinejad, J. Speyer, and G. Pottie, "A distributed scheme for integrated predictive dynamic channel and power allocation in cellular radio networks," in IEEE Global Telecommunications Conference, vol. 6, San Antonio, USA, 2001, pp. 3623-3627.

[6] A. Subramanian and A. H. Sayed, "A robust power and rate control method for state-delayed wireless networks," Automatica, vol. 41, no. 11, pp. 1917-1924, 2005.

[7] M. Xiao, N. B. Shroff, and E. K. Chong, "A utility-based powercontrol scheme in wireless cellular systems," IEEE/ACM Transactions on Networking, vol. 11, no. 2, pp. 210-221, 2003.

[8] B. Muhammad and A. Mohammed, "Uplink closed loop power control for LTE system," in IEEE International Conference on Emerging Technologies, Islamabad, Pakistan, 2010, pp. 88-93.

[9] R. Mullner, C. Ball, K. Ivanov, J. Lienhart, and P. Hric, "Contrasting open-loop and closed-loop power control performance in UTRAN LTE uplink by UE trace analysis," in IEEE International Conference on Communications, Dresden, Germany, 2009, pp. 1-6.

[10] L. Song and J. Shen, Evolved cellular network planning and optimization for UMTS and LTE. CRC Press, 2010.

[11] TS 36.213, Physical layer procedures, 3GPP (Third Generation Parternship Program), 2011.

[12] A. Bemporad, M. Morari, V. Dua, and E. Pistikopoulos, "The explicit linear quadratic regulator for constrained systems," Automatica, vol. 38, no. 1, pp. 3-20, 2002.

[13] P. Gutman, "A linear programming regulator applied to hydroelectric reservoir level control," Automatica, vol. 22, no. 5, pp. 533-541, 1986.

[14] P. Ogren, E. Fiorelli, and N. E. Leonard, "Cooperative control of mobile sensor networks: Adaptive gradient climbing in a distributed environment," IEEE Transactions on Automatic Control, vol. 49, no. 8, pp. 1292-1302, 2004.

[15] E. Rimon and D. Koditschek, "Exact robot navigation using artificial potential functions," IEEE Transactions on Robotics and Automation, vol. 8 , no. 5 , pp. 501-518, 1992.

[16] C. Ionete, A. Cela, and M. B. Gaid, "Controllability and observability of input/output delayed discrete systems," in IEEE American Control Conference, 2006, pp. 3513-3518.

[17] J. Nilsson, "Real-time control systems with delays," Ph.D. dissertation, Department of Automatic Control, Lund Institute of Technology, 1998.

[18] T. Rappaport, Wireless Communications: Principles and Practice. Prentice Hall, 2002.

[19] S. Yang, OFDMA system analysis and design. Artech House Publishers, 2010.

[20] F. Khan, LTE for $4 G$ mobile broadband: air interface technologies and performance. Cambridge University Press, 2009.

[21] I. T. Stefania Sesia and M. Baker, LTE - The UMTS Long Term Evolution From Theory to Practice. John Wiley \& Sons Ltd, 2009.

[22] R. Kreher and K. Gaenger, LTE Signaling: Troubleshooting and Optimization. John Wiley \& Sons Ltd, 2011.

[23] E. Dahlman, S. Parkvall, and J. Skold, 4G: LTE/LTE-Advanced for Mobile Broadband. Academic Press, 2011.

[24] Motorola, "Long term evolution (LTE): Overview of LTE air-interface," Technical white paper, 2007.

[25] Z. Jiang and Y. Wang, "A converse Lyapunov theorem for discrete-time systems with disturbances," Systems \& Control Letters, vol. 45, no. 1, pp. 49-58, 2002.

[26] K. Astrom and B. Wittenmark, Computer-controlled systems: theory and design. Dover Publications, 2011.

[27] S. Elaydi, An Introduction to Difference Equations. Springer, 2005.

[28] J. Lei, R. Yates, L. Greenstein, and H. Liu, "Wireless link SNR mapping onto an indoor testbed," in Testbeds and Research Infrastructures for the Development of Networks and Communities, Trento, Italy, 2005, pp. 130 -135 .

[29] C. Castellanos, D. Villa, C. Rosa, K. Pedersen, F. Calabrese, P. Michaelsen, and J. Michel, "Performance of uplink fractional power control in UTRAN LTE," in IEEE Vehicular Technology Conference, 2008, pp. 2517-2521. 